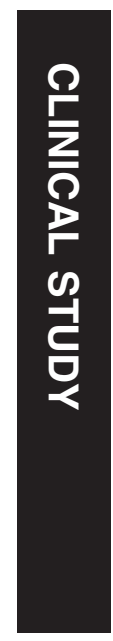

\title{
The psychological well-being and appearance concerns of patients presenting with ptosis
}

${ }^{1}$ Faculty of Health and Applied Sciences, Centre for Appearance Research, University of the West of England, Bristol, UK

${ }^{2}$ Bristol Eye Hospital, Bristol, UK

Correspondence: HS Richards, Centre for Appearance Research, University of the West of England, Coldharbour Lanes, Bristol,

BS16 1QY, UK

Tel: +44 (0)7735425188;

E-mail: hollie.richards@

bristol.ac.uk

Received: 10 June 2013 Accepted in revised form: 24 October 2013

Published online:

20 December 2013

\begin{abstract}
Purpose Ptosis affects both the function and appearance of the eyes and face, because of drooping of the eyelids. Previous research has focused on functional impairment; however, similar appearance altering eye conditions have been demonstrated to have major impacts on psychosocial functioning. Methods This study examines the psychological well-being and appearance concerns of patients presenting with ptosis recruited from Bristol Eye Hospital using validated questionnaires focusing on demographics and psychosocial factors before surgery.

Results Patients reported higher levels of anxiety, depression, and appearance concern than population norms, with scores comparable to previous research examining the impact of other disfiguring eye conditions. Gender differences were observed, with almost half of females experiencing significant psychosocial issues related to appearance concern about their ptosis compared with $20 \%$ of males. Linear regressions revealed that the main predictor of poor adjustment to the appearance of ptosis was the level of concern an individual had regarding the negative perceptions of other people. These results implicate psychosocial factors and demographics as predictors of the development of appearancerelated distress and compromised psychological well-being for patients with ptosis who are currently seeking surgery. Conclusions This study provides clinicians with a greater understanding of the concerns of affected patients, and treatment motivations. The findings may also inform the development of patient reported outcome measures and
\end{abstract}

HS Richards' ${ }^{1}$ E Jenkinson', N Rumsey ${ }^{1}$, P White ${ }^{1}$, H Garrott ${ }^{2}$, H Herbert ${ }^{2}$, F Kalapesi ${ }^{2}$ and RA Harrad ${ }^{2}$

support for patients, which targets the psychosocial impact of the condition.

Eye (2014) 28, 296-302; doi:10.1038/eye.2013.264; published online 20 December 2013

Keywords: ptosis; psychological distress; outpatients

\section{Introduction}

Ptosis is the drooping of one or both eyelids, affecting both appearance and function of the eyes. An estimated $11.5 \%$ of adults in the United Kingdom over the age of 55 years have ptosis, ${ }^{1}$ most commonly resulting from either stretching of the levator muscle tendon or separation of the tendon from the tarsus. Ptosis can cause visual impairments, loss of peripheral vision and blurred vision. Ptosis also results in altered facial appearance, with patients reporting that they looked 'tired'. ${ }^{2}$ Following ptosis surgery, patients ranked eye and eyelid appearance as the most important post-operative change after improved visual field. ${ }^{3}$

Indeed, the psychological impact of an altered facial appearance coupled with physical challenges can have both 'interpersonal' and 'intrapersonal' consequences. ${ }^{4}$ Reactions of others to people with appearance altering eye conditions can be stigmatizing. Evaluations of photographs of people with esotropic strabismus resulted in more negative ratings of personality traits such as intelligence, communication skills, and attentiveness than photographs of people without the condition. ${ }^{5}$ Patients with strabismus, ptosis, or dermatatochalasis were also viewed as less dateable, less attractive, less likeable, and less successful in studies, in which people were asked to rate photographs and headshots of 
potential dates. ${ }^{6,7}$ Difficulties obtaining employment ${ }^{8,9}$ and career progression and promotion within the US Army ${ }^{10}$ have also been highlighted by the research.

Psychosocial distress has been shown to be associated with the presence of an eye condition. In a study of patients with strabismus, Jackson et al ${ }^{11}$ found higher levels of social anxiety and avoidance compared with general population norms. In studies of patients with a range of visible differences affecting the eye, social avoidance and distress related to appearance were reported by $45 \%$ of patients. ${ }^{12}$ Recent research from a sample of 98 patients with disfiguring eye conditions, including some ptosis patients, showed that although most patients appeared to be coping well when compared with clinical norms derived from previous research, $22 \%$ scored above the threshold for clinical anxiety. Over $40 \%$ of patients were experiencing high levels of distress related to their looks as measured by the Derriford Appearance Scale (DAS 24). ${ }^{13}$

These findings, triangulated with studies examining the psychosocial impact of other visible differences such as those caused by burns injuries, cleft lip and palate, and skin conditions, suggest that the presence of an appearance altering condition does not necessarily result in poor psychological well-being. Psychological studies have shown that many demographic, social, environmental, and psychological factors may mediate coping and adjustment in patients. ${ }^{14}$ In patients with eye conditions, being older, male, and married or living with a partner was related to higher levels of psychological well-being. ${ }^{13}$ Physically and psychologically, a less visible condition, greater ability to disguise the affected area, a more positive evaluation of their own overall appearance, less engagement in comparing themselves with others, greater feelings of being accepted by others, appearance being less important to their self concept, and a smaller discrepancy between the person's ideal and actual appearance were all related to better psychological adjustment. ${ }^{13}$

However, the psychosocial issues associated specifically with ptosis have not yet been fully examined by researchers. Previous studies have focused on the impact on patients' vision and function as a consequence of the condition, but neglected to investigate patients' psychological well-being and concerns about the appearance of their eyes. In light of previous research suggesting that disfiguring eye conditions can have negative psychological and social impacts and that psychological factors may be mediating patients' satisfaction with surgical outcomes, ${ }^{3}$ these factors require further investigation to inform clinical practice.

This study aims to examine and quantify the psychological well-being of adults with ptosis through the use of standardized psychosocial measures. This research builds on previous studies of the consequences of disfiguring eye conditions (ie, 13) by focusing exclusively on patients diagnosed with ptosis and examines the extent to which this condition impacts on patients' well-being, specifically in relation to psychological and social functioning. This study measures demographic and psychosocial factors that may influence psychological adjustment to ptosis and investigates the relationships between these variables. This research was undertaken in order to inform clinicians and health-care professionals about ways in which ptosis may be affecting patients, and to inform evidence-based practice, which addresses identified needs.

\section{Materials and methods}

A total of 78 adult patients attending the Bristol Eye Hospital between 2008 and 2012 were invited to take part. Patients were included if they were over 18 years, presented with unilateral or bilateral ptosis of any aetiology, had a fluent understanding of English and had been listed for surgery. The majority of patients were listed for surgery on the basis of visual symptoms, not solely because of concerns regarding their appearance. Eligible patients were included regardless of additional conditions or visual acuity (VA). Fifty-nine out of sixtyone patients had VA 6/12 or better in both eyes. One patient had Counting Fingers vision in one eye and one patient had VA of 6/60 and 3/60. All participants completed measures before any corrective surgery.

\section{Materials}

Four validated psychological measures were administered to all participants. Patient well-being was assessed using the short form of the DAS 24 and by the Hospital Anxiety and Depression Scale (HADS). The Valence of Appearance scale (CARVAL) was used to determine the extent to which patients positively or negatively evaluated their own appearance. The Brief Version of the Fear of Negative Evaluation Scale (FNE) was administered to determine the extent to which patients were concerned about how they are perceived and judged by other people.

- The DAS $24^{15}$ is a 24-item scale, which measures levels of distress and loss of function because of perceived problems with appearance. ${ }^{16}$ Scores range from 11 to 96 with increasing scores indicating poorer adjustment. The scale has previously been used in a similar ophthalmic population. ${ }^{13}$ The scale demonstrates good internal reliability (Cronbach's alpha $=0.92)$; good 6-month test-retest reliability in a clinical population $(r=0.82)$, and good 3-month test-retest in a general population $(r=0.88) .{ }^{15}$ 
- The HADS ${ }^{17}$ identifies possible and probable cases of anxiety and depression among non-psychiatric hospital patients. It consists of a depression subscale and anxiety subscale with scores ranging from 0 to 21 for each scale with higher scores indicating more severe levels of distress. The accepted threshold score for the occurrence of clinical levels of anxiety or depression is 11. The HADS has been used extensively, both clinically and for research purposes, and has demonstrated good internal consistency and good concurrent validity in comparison to other similar measures (range of $r=0.60-0.80$ ). ${ }^{18}$

- The Brief Version of the FNE consists of 12 items describing fearful or worrying cognitions in relation to the perceived opinions of others. ${ }^{19}$ The scale measures apprehension about the evaluations made by others and distress caused by these perceived evaluations. Scores range from 12 to 60 , with higher scores indicating greater fear of negative evaluation. FNE demonstrates good internal consistency (coefficient alpha $=0.90$ ) and high 4-week test-retest reliability within a student population $(r=0.75) .{ }^{19}$

- The CARVAL ${ }^{20}$ is a six-item measure, which assesses the extent to which individuals evaluate their appearance in a positive or negative way. Scores range from 6 to 36, with higher scores indicating more negative evaluations. The CARVAL demonstrates good test-retest reliability at 3 months $(r=0.95)$ and good internal consistency (coefficient alpha $=0.89$ ).

\section{Statistical analysis}

In order to address the aims of the study, inferential statistical analyses were performed. Data integrity checks were performed before analysis and data were screened for potential influential response patterns, which might otherwise unduly contribute to errors in statistical inference. No such unusual patterns were detected. One participant did not complete the DAS 24 (effective sample size $N=60$ ) and four did not complete the HADS (effective sample size $N=57$ ). Analyses were undertaken using all available data for each analysis. Differences between groups were examined using standard parametric tests (independent samples $t$-test, ANOVA) and relationships between scale data were examined using standard parametric tests (Pearson's correlation coefficient, multiple linear regressions). A robust assessment of the statistical assumptions underpinning these techniques was performed. These tests of assumptions did not cast doubt on the appropriateness of the data analysis plan. Two-sided tests were used to evaluate the significance of correlations and two group comparisons. Significance was judged against a nominal level of alpha $=0.05$. The data were analysed using SPSS version 19 (SPSS Inc., Chicago, IL, USA).

\section{Statement of ethics}

We certify that all applicable institutional and governmental regulations concerning the ethical use of human volunteers were followed during this research.

\section{Results}

Sixty-one patients took part in the study. The sample comprised $n=35$ females and 26 males, with an age range of $18-84($ median $=64$, mean $=61.6, \mathrm{SD}=15.3)$ years. Twenty-one participants were aged $18-60$ years, 20 were aged 60-70 years and 20 were aged over 70 years. All measures displayed good internal consistency, with Cronbach's alpha scores of greater than 0.7.

Table 1 displays the mean scores for all variables by gender. Females had higher mean values on all measures than males. Analysis using the independent samples $t$-test showed that the difference in means achieve statistical significance on Fear of Negative Evaluation $(P=0.001)$, and on CARVAL $(P=0.001)$, but not on DAS $24(P=0.074)$, HAD Anxiety $(P=0.083)$, or HAD Depression $(P=0.305)$. Extending the analysis to include age as a covariate indicated that there were significant gender differences for HAD Anxiety $(P=0.013)$, HAD Depression $(P=0.045)$, CARVAL $(P=0.002)$, FNE $(P=0.014)$, and DAS $24(P=0.006)$.

Of the 34 female patients who completed HADS, 35.2\% scored 11 or higher on the anxiety domain indicating moderate or severe anxiety, and 8.8\% similarly scored above the threshold for moderate depression. Of the 23 male patients who completed HADS, 21.7\% scored above the threshold for moderate anxiety with $12.9 \%$ recording scores indicative of moderate or severe depression.

The mean DAS 24 score for women was $38.4(N=36$, $\mathrm{SD}=14.2)$ and $32.0(N=25, \mathrm{SD}=12.0)$ for men.

The relatively high standard deviation in participant DAS 24 scores is indicative of considerable variation between participants, with some experiencing moderate levels of appearance-related psychosocial effects. In all,

Table 1 Summary statistics for psychological measures

\begin{tabular}{|c|c|c|c|c|c|c|}
\hline \multirow[t]{2}{*}{ Measure } & \multicolumn{2}{|c|}{ All $(\mathrm{n}=61)$} & \multicolumn{2}{|c|}{ Male $(\mathrm{n}=26)$} & \multicolumn{2}{|c|}{ Female $(\mathrm{n}=35)$} \\
\hline & Mean & $S D$ & Mean & $S D$ & Mean & $S D$ \\
\hline DAS 24 & 35.72 & 13.62 & 32.0 & 12.0 & 38.37 & 14.23 \\
\hline HADS Anxiety & 7.93 & 4.88 & 6.57 & 4.32 & 8.85 & 5.09 \\
\hline HADS Depression & 4.74 & 3.64 & 4.13 & 4.21 & 5.15 & 3.19 \\
\hline CARVAL & 19.76 & 7.48 & 16.32 & 4.24 & 22.38 & 8.37 \\
\hline FNE & 34.35 & 10.86 & 28.24 & 9.31 & 38.12 & 10.11 \\
\hline
\end{tabular}


$72 \%$ of females and $68 \%$ of males reported high levels of concern, scoring over 30 on the DAS 24. In addition, $48.9 \%$ of female patients scored above the threshold for significant issues related to appearance concerns (over 40), compared with $20 \%$ of male patients.

These mean values are comparable with findings from other groups of patients with eye conditions affecting appearance. Table 2 summarizes this information, also displaying data from non-clinical populations.

For females, age was significantly negatively correlated with DAS $24(r=-0.638, P<0.001)$, CARVAL $(r=-0.486, P=0.004)$, FNE $(r=-0.521, P=0.002)$, and HAD Anxiety $(r=-0.554, P=0.001)$ but not HAD Depression $(r=-0.211, P=0.230)$. For males, there were no significant correlations between age and any of the variables (DAS $24(P=0.192)$, CARVAL $(P=0.623)$, FNE $(P=0.358)$, HAD Anxiety $(P=0.449)$ or HAD Depression $(P=0.195))$.

For the total sample, scores on the DAS 24 were significantly correlated with HAD Anxiety $(r=0.688$, $P<0.001)$, HAD Depression $(r=0.575, P<0.001)$, FNE $(r=0.692, P<0.001)$, and CARVAL $(r=0.467, P<0.001)$. For females, DAS 24 was significantly correlated with HAD Anxiety $(r=0.666, P<0.001)$, HAD Depression $(r=0.587, P<0.001)$, FNE $(r=0.700, P<0.001)$, and CARVAL $(r=0.495, P=0.003)$. For males, DAS 24 was significantly correlated with HAD Anxiety $(r=0.683$, $P<0.001)$, HAD Depression $(r=0.565, P<0.001)$, FNE $(r=0.664, P<0.001)$ but not with CARVAL $(r=0.244$, $P=0.239)$.

Linear regressions were conducted to determine which factors influenced psychosocial adjustment to appearance concern as measured by DAS 24 and generalized anxiety as measured by HAD Anxiety. In all, $53.4 \%$ of the sample variation in DAS 24 scores was explained by a regression model including age, gender, CARVAL, and FNE as predictor variables $(P<0.001)$. However, FNE significantly mediated the effect of CARVAL on DAS 24 and FNE was the dominant effect as shown in Table 3a. The second regression (Table 3b) used the same structural relationship to model generalized anxiety as measured by HAD Anxiety. In this model, $33.4 \%$ of the sample variation in HAD Anxiety scores was explained by a model including age, gender, CARVAL, and FNE as predictors $(P<0.001)$. However, as with DAS 24, FNE significantly mediated the CARVAL effect and FNE was the only significant variable $(P<0.05)$ as shown in Table $3 b$.

\section{Discussion}

Building on previous research of patients with a range of disfiguring eye conditions, this study aimed to explore in more detail the potential psychosocial impact and appearance-related concern of patients with ptosis. Patients reported appearance-related distress and dysfunction because of their ptosis, with mean scores higher than the general population and comparable with previous studies of ophthalmic outpatient clinic attendees, ${ }^{13,11}$ with $48.9 \%$ of female patients experiencing elevated levels of distress. This confirms that outpatient attendees with ptosis experience appearance-related distress and maladjustment to a similar extent as patient groups with strabismus and other appearance altering eye conditions. Therefore, as identified by James et al, ${ }^{13}$ appearance-related distress may be a motivating factor in seeking surgery, with patients expectations of the benefits of surgery focusing on the alleviation of appearance-related distress as well as functional aspects. As a corollary, concerns about appearance should be considered in the development of 'patient reported outcome measures (PROMS)' for this population.

Levels of anxiety and depression were also higher than non-clinical population norms ${ }^{21}$ and higher than preoperative strabismus patients and patients with other eye conditions. ${ }^{11,13}$ These mean scores were below clinical thresholds, indicating that as a whole this was not a clinically depressed or anxious population. However, $23.5 \%$ of female patients and $21.7 \%$ of male patients scored above the threshold for severe anxiety (score of $15+)$, indicating that a proportion of the patients were experiencing significant levels of anxiety. Gender and age effects on psychosocial adjustment to ptosis were observed in line with previous studies, ${ }^{22,13}$ with women and younger people displaying higher levels of concern and reporting more extensive psychosocial impact. There were higher levels of anxiety observed in female than in male participants with $35.2 \%$ of women scoring above the threshold for moderate anxiety and nearly half of all females experiencing considerable levels of appearancerelated psychosocial affects because of ptosis. In addition, as FNE scores explain the largest proportion of the variance in DAS 24 and HADS anxiety, this may indicate that the higher levels of clinical anxiety and appearance-related dysfunction and distress observed in female patients are mediated by concerns about being judged negatively by others; a variable potentially amenable to change by psychological intervention.

Overall, this group was equally as anxious, depressed, and distressed about the appearance of their eye condition when compared with populations with other appearance altering eye conditions, and on average were more likely to be anxious, depressed, and concerned about their appearance than the general population. This is a noteworthy finding given that the majority of the participants in this study were over 60 years, when older adulthood is often (erroneously) cited as providing 
Table 3a Regression model for DAS 24

\begin{tabular}{|c|c|c|c|c|c|}
\hline & \multicolumn{2}{|c|}{$\begin{array}{l}\text { Unstandardized } \\
\text { coefficients }\end{array}$} & \multirow{2}{*}{$\begin{array}{c}\begin{array}{c}\text { Standardized } \\
\text { coefficients }\end{array} \\
\text { Beta }\end{array}$} & \multirow[t]{2}{*}{$\mathrm{T}$} & \multirow[t]{2}{*}{ Sig. } \\
\hline & B & $S E$ & & & \\
\hline (Constant) & 20.43 & 8.88 & & 2.300 & 0.026 \\
\hline Age & -0.193 & 0.096 & -0.215 & -2.014 & 0.050 \\
\hline CARVAL & 0.078 & 0.234 & 0.044 & 0.336 & 0.738 \\
\hline FNE & 0.815 & 0.171 & 0.640 & 4.771 & $<0.001$ \\
\hline Gender & -3.401 & 3.168 & -0.121 & -1.074 & 0.288 \\
\hline
\end{tabular}

Gender dummy variable coded, male $=0$; female $=1$.

Table 3b Multiple regression model for HAD Anxiety

\begin{tabular}{|c|c|c|c|c|c|}
\hline & \multicolumn{2}{|c|}{$\begin{array}{l}\text { Unstandardized } \\
\text { coefficients }\end{array}$} & \multirow{2}{*}{$\begin{array}{c}\begin{array}{c}\text { Standardized } \\
\text { coefficients }\end{array} \\
\text { Beta }\end{array}$} & \multirow[t]{2}{*}{$\mathrm{T}$} & \multirow[t]{2}{*}{ Sig. } \\
\hline & B & $S E$ & & & \\
\hline (Constant) & 5.471 & 3.632 & & 1.506 & 0.138 \\
\hline Age & -0.076 & 0.039 & -0.245 & -1.927 & 0.060 \\
\hline CARVAL & 0.081 & 0.096 & 0.132 & 0.845 & 0.402 \\
\hline FNE & 0.146 & 0.070 & 0.334 & 2.083 & 0.042 \\
\hline Gender & 0.272 & 1.296 & 0.028 & 0.210 & 0.834 \\
\hline
\end{tabular}

Gender dummy variable coded, male $=0$; female $=1$.

respite from concerns about appearance. ${ }^{23}$ This study provides further evidence that older patients are not immune to appearance-related distress and dysfunction, even if advancing age can be viewed as a protective factor. Similarly, in relation to gender differences, although women reported poorer outcomes on average, some men also reported high levels of concern, indicating no simple relationship between demographics and psychological adjustment to ptosis. This variability has been noted in previous studies. ${ }^{13}$ The findings here serve to strengthen the notion that although gender and age may have some bearing on the psychological well-being of adults with disfiguring eye conditions, assessment of patient needs should consider individual differences, rather than assumptions about likely levels of distress based on gender and age.

Consistent with previous research, psychological rather than demographic factors were most predictive of patient levels of appearance-related distress and dysfunction. Concern about being negatively evaluated by others, a high subjective value placed on appearance, and high levels of anxiety were implicated in higher levels of distress about ptosis. Put simply, those for whom appearance is of greater concern within their selfevaluations may cope less well with the challenges of an appearance altering eye condition. Indeed, this finding follows previous work in the field of appearance altering 
conditions, in which fear of rejection, social avoidance, and social stigma of looking 'visibly different' 24 have been implicated in the development of anxiety, depression, and social avoidance. ${ }^{14,25,26}$ Stigma occurs when an individual is perceived as deviating from social norms, ${ }^{27}$ including what is considered by a society to be normal appearance. ${ }^{28}$ Facial appearance, and specifically eyes and eyelids, strongly influence the impression an individual will form about a person, ${ }^{29,30}$ and these negative perceptions tend to be complex. ${ }^{31}$ Eyes that appear symmetrical and open adhere to perceived social norms of health and attractiveness, and deviation from this can lead to stigma and stigmatizing behaviours and cognitions. Previous research suggests patients with eye conditions are similarly affected, and highlights the farreaching consequences of the associated objective and subjective stigma, ${ }^{32}$ which can impact on psychosocial functioning including relationship difficulty ${ }^{33}$ and being deemed less attractive to others. ${ }^{7}$

This study is limited by focusing solely on the psychosocial impact of ptosis as experienced by patients in contact with the National Health Service, and all patients were in the process of gathering information about or seeking treatment, and as such may not be representative of those who do not seek treatment. In addition, data relating to the length of time patients had been experiencing ptosis was not included in analysis.

Nevertheless, this is the first study to examine the psychological well-being of pre-operative ptosis patients and provides an insight into the needs of this patient group. Anxiety, depression, and distress about appearance were found to be comparable to patients with other appearance altering eye conditions and higher than the general population norms. These concerns may be stronger motivators in seeking surgical consultations than the functional aspects of the condition. Therefore, this study has made significant steps towards the development of PROMS for oculofacial visible differences.

This study also highlights the need for more support and guidance for patients in their decision making around surgery, management of expectations of the clinical outcomes, and tailored follow-up care from ophthalmologists, nurses, and psychologists to ensure patient satisfaction and well-being. For the minority of patients for whom appearance distress and anxiety is debilitating, referral to specialist psychological support offered by an appropriately trained health, clinical, or counselling psychologist as an alternative or adjunct to surgical intervention is required. Psychological interventions such as CBT could be targeted to help patients reduce their anxiety, manage their fear of negative evaluation by others, and manage concerns about their appearance more effectively. ${ }^{34,35}$ However, a lack of research to guide recommendations about the most appropriate form of psychological intervention for patients with appearance-related distress was highlighted in a recent systematic review. ${ }^{36}$ Future research is required to further enhance understanding of the needs of these patients gained from this study and to examine the effectiveness of surgical and psychosocial interventions in optimizing psychological well-being. Researchers from The Centre for Appearance Research (CAR), UWE, and the Bristol Eye Hospital are in the process of interviewing patients and gathering qualitative data in order to gain a deeper insight into the needs of patients with ptosis.

\section{Summary}

\section{What was known before}

- Disfiguring eye conditions can have a negative psychological impact on many patients. This can include the development of anxiety, social avoidance, and concealing behaviours.

- How well patients cope is not well predicted by the extent or severity of their eye condition. Previous research has identified demographic factors associated with higher levels of positive adjustment, with older adults and men reporting lower levels of anxiety, depression, and appearance concerns than young adults and women. However, psychological factors may have a greater impact on coping, with those who place a high value on appearance overall, and who compare themselves to appearance ideals, most at risk of experiencing distress in relation to their condition.

- The psychological well-being of ptosis patients remained under research, and whether the findings from studies with patients with other disfiguring eye conditions (such as strabismus and thyroid eye disease) could be generalized to this patient group was unclear.

\section{What this study adds}

- The majority of patients with ptosis cope well with the psychosocial challenges of their condition.

- However, the data suggests that patients with ptosis are likely to report higher levels of anxiety, depression, and concern about their appearance than the general population.

- Consistent with previous studies, being older and male, less anxious, with less valued placed on appearance and a low level of concern about being negatively evaluated by others was predictive of adjustment to the impact of ptosis.

- These findings can be used to support patients with ptosis and provide clinicians with a starting point from which to design interventions targeting the psychosocial impact of the condition.

\section{Conflict of interest}

The authors declare no conflict of interest. 


\section{Acknowledgements}

We thank all of the patients from Bristol Eye Hospital who agreed to take part in this study, as well as the Bristol Eye Hospital team for their assistance in the data collection process. This project was supported by funding from British Oculoplastic Surgery Society Collin Prize awarded to Richard Harrad.

\section{References}

1 Sridharan GV, Tallis RC, Leatherbarrow B, Forman WM. Acommunity survey of ptosis of the eyelid and pupil size of elderly people. Age Ageing 1995; 24: 21-24.

2 Finsterer J. Ptosis: causes, presentation, and management. Aesthetic Plast Surg 2003; 27: 193-204.

3 Federici TJ, Meyer DR, Lininger LL. Correlation of the vision-related functional impairment associated with blepharoptosis and the impact on blepharoptosis surgery. Ophthalmology 1999; 106: 1705-1712.

4 Partridge J, Pearson. A. Don't worry it's the inside that counts. Psychologist 2008; 21(6): 490-491.

5 Olitsky SE, Sudesh S, Graziano A, Hamblen J, Brooks SE, Saha SH. The negative psychosocial impact of strabismus in adults. J AAPOS 1999; 3: 209-211.

6 Bullock JD, Warwar RE, Bienenfeld DG, Marciniszyn SL, Markert RJ. Psychosocial implications of blepharoptosis and dermatochalasis. Trans Am Ophthalmol Soc 2001; 99: 65-72.

7 Mojon-Azzi SM, Potnik W, Mojon DS. Opinions of dating agents about strabismic subjects' ability to find a partner. Br J Ophthalmol 2008; 92: 765-769.

8 Coats DK, Paysse EA, Towler AJ, Dipboye RL. Impact of obvious uncorrected horizontal strabismus on ability to obtain employment. Ophthalmology 2000; 107: 402-405.

9 Mojon-Azzi SM, Mojon DS. Strabismus and employment: the opinion of headhunters. Acta Ophthalmologica 2009; 87: 784-788.

10 Goff MJ, Suhr AW, Ward JA, Croley JK, O’Hara MA. Effect of adult strabismus on rating of official U. S. Army photographs. J AAPOS 2006; 10: 400-403.

11 Jackson S, Harrad R, Morris M, Rumsey N. The psychosocial benefits of corrective surgery for adults with strabismus. Br J Ophthalmol 2006; 90: 883-888.

12 Clarke A, Rumsey N, Collin JR, Wyn-Williams M. Psychosocial distress associated with disfiguring eye conditions. Eye 2003; 17: 35-40.

13 James H, Jenkinson E, Harrad R, Ezra D, Newman S \& Members of the Appearance Research Collaboration (ARC). Appearance concerns in ophthalmic patients. Eye 2011; 25: 1039-1044.

14 Rumsey N, Harcourt D. The Psychology of Appearance. Open University Press: Maidenhead, 2005.

15 Carr T, Moss TP, Harris DL. The DAS 24: A short form of the Derriford Appearance Scale (DAS59) to measure individual responses to living with problems of appearance. $\mathrm{Br} \mathrm{J} \mathrm{Health}$ Psychol 2005; 10: 285-298.
16 Moss TP. The relationship between objective and subjective ratings of disfigurement severity, and psychological adjustment. Body Image 2005; 2: 151-159.

17 Zigmond AS, Snaith RP. The Hospital Anxiety and Depression Scale. Acta Psychiatrica Scandinavica 1983; 67: 361-370.

18 Bjelland I, Dahl AA, Haug TT, Neckelmann D. The validity of the Hospital Anxiety and Depression Scale - An updated literature review. J Psychosomatic Res 2002; 52: 69-77.

19 Leary MR. A brief version of the Fear of Negative Evaluation Scale. Pers Soc Psychol Bull 1983; 9: 371-375.

20 Moss TP, Rosser BA. The moderated relationship of appearance valence on appearance self consciousness: development and testing of new measures of appearance schema components. PLoS One 2012; 7(11): e50605.

21 Crawford JR, Henry JD, Crombie C, Taylor EP. Normative data for the HADS from a large non-clinical sample. Br J Clin Psychol 2001; 40: 429-434.

22 Harris D, Carr A. Prevalence of concer about physical appearance in the general population. Br J Plast Surg 2001; 54: 223-226.

23 Baker L, Gringart E. Appearance in later life. In: Rumsey N, Harcourt D (eds). The Handbook of the Psychology of Appearance. Oxford University Press: Oxford, 2012.

24 Lansdown R, Rumsey N, Bradbury E. Visibly Different: Coping with Disfigurement. Butterworth-Heinemann: Oxford, 1997.

25 Rumsey N, Clarke A, White P. Exploring the psychosocial concerns of out-patients with disfiguring conditions. J Wound Care 2003; 12: 247-252.

26 Ong J, Clarke A, White P, Johnson M, Withey S, Butler PEM. Does severity predict distress? The relationship between subjective and objective measures of appearance and psychological adjustment, during treatment for facial lipoatrophy. Body Image 2007; 4: 239-248.

27 Goffman E. Stigma. Penguin: London, 1963.

28 Link BG, Phelan JC. Conceptualising stigma. Ann Rev Sociol 2011; 27: 363-385.

29 Zebrowitz LA. Reading Faces. Boulder. Westview Press: Colorado, 1998.

30 Liggett J. The Human Face. Stein \& Day: New York, 1974.

31 Stock NM, Whale K, Jenkinson E, Rumsey N, Fox F. Young people's perceptions of visible difference. Diversity Equality Health Care 2013; 10: 41-51.

32 Durnian JM, Noonan CP, Marsh IB. The psychosocial effects of adult strabismus: a review. Br J Ophthalmol 2011; 95: 450-453.

33 Satterfield D, Keltner JL, Morrison TL. Psychosocial aspects of strabismus study. Arch Ophthalmol 1993; 111: 1100-1105.

34 Clarke A, Thompson A, Rumsey N, Jenkinson E, Newell RJ. CBT for Appearance Anxiety. Wiley Blackwell, 2013.

35 Jenkinson E. Therapeutic interventions for people with a visible difference: Evidence of effectiveness. In: Rumsey N, Harcourt D (eds) Oxford Handbook of the Psychology of Appearance. Oxford University Press: Oxford, 2012.

36 Bessell A, Moss TP. Evaluating the effectiveness of psychosocial interventions for individuals with visible differences: A Systematic Review of the empirical literature. Body Image 2007; 4: 227-238. 